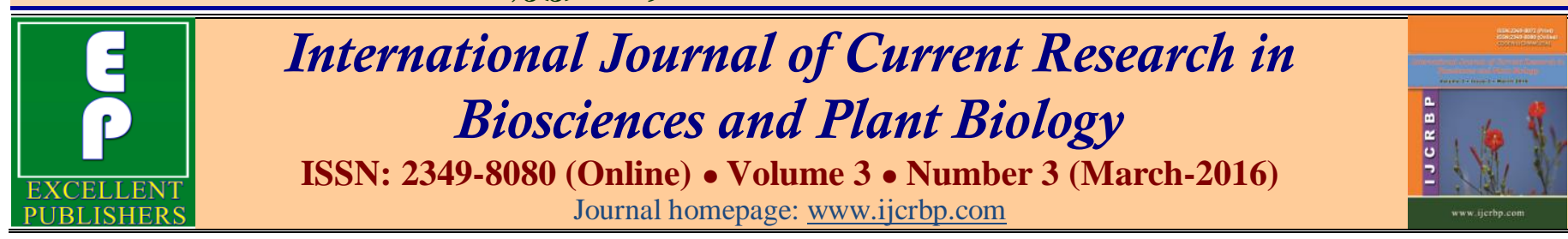

\title{
Diversity of Invasive Alien Plants in Soor Sarovar Bird Sanctuary (SSBS), Keetham, Agra, India
}

\author{
Vijay V. Wagh* \\ Plant Diversity Systematics and Herbarium Division, CSIR-National Botanical Research Institute, Lucknow- 226 oo1, Uttar \\ Pradesh, India \\ *Corresponding author.
}

\begin{abstract}
A bstract
Present study provides the comprehensive list of invasive alien plants of Soor Sarovar Bird Sanctuary, Keetham, Agra. In study area total of 76 species under 67 genera and 35 families have been found as invasive alien plant. Of total only 09 species have been found introduced intentionally, while the other species introduced unintentionally. In terms of nativity the majority of invasive plants reported from American continent $(61 \%)$. The life form category shows that 56 species are herbs, 11 species of shrubs, 4 species of climbers, 4 species of trees and only one liana species. The invasive alien species possess serious threat to local biodiversity, effects crop production and human health. Therefore systematic study about the invasive plant species is required.
\end{abstract}

\section{Article Info}

Accepted: 04 March 2016

Available Online: 06 March 2016

\section{Ke ywords}

Biodiversity

Invasive plants

Soor Sarovar Bird Sanctuary

Threat

\section{Introduction}

Alien species are non-native or exotic organisms that occur outside their natural adapted ranges and dispersal potential (McGeoch et al., 2010). These invasive species are widely distributed in all kinds of ecosystems throughout the world and include all categories of living organisms. Nevertheless, plants, mammals, and insects comprise the most common types of invasive alien species in terrestrial environments (Raghubanshi et al., 2005). An important requirement for successful colonization of invaders is open habitat with reduced competition. Generally, the microsites created by grazing may be occupied by invader species (Singh, 1976; Sinha, 1976; Sawarker, 1984).

Invasive alien plant caused an impact worldwide (Mooney and Hobbs, 2000), there are still many regions such as Asia and Neighbouring region in the world where basic information on naturalized plant taxa and plant invasions is lacking (Corlett, 1988; Enmoto, 1999;
Meyer, 2000). Database establishment of naturalized species is the first step in the development of invasion biology, and will also serve as a stepping stone for further detailed studies on the biology and impact of individual species (Wu et al., 2004). Naturalization has been recognized as the first phase of biological invasions. A naturalized species is the species that can consistently reproduce and sustain populations over many generations without direct intervention by humans (Richardson et al., 2000; Pyšek et al., 2002). Many invasive plant species cause economic and/or environmental damage, and referred to as alien pests or weeds (Richardson et al., 2000).

In India Invasive alien plants became a issue of great concern that affects the local biodiversity of the country. These species were introduced in India accidentally or deliberately mostly for fodder and ornamental purpose. Due to its growing concern and harmful effects on the biodiversity several studies has been carried out in different regions of India out of that some important are, 
impact of Lantana camara on the biodiversity of Melghat Tiger Reserve, Maharashtra by Sawarker (1984), Pandey and Parmar (1994) worked on the exotic flora of Rahasthan. Kshirsagar (2005) made a very interesting study on the origin, present status and distribution of exotic plants of South Gujarat, Alien flora of Doon valley has been done by Negi and Hajra (2007), Sekar (2012) made a comprehensive study on the invasive alien plants of Indian Himalayan region, Das and Durah (2013) worked on invasive alien plant of Johrat, Assam, Srivastava et al. (2014) and Wagh and Jain (2015) made study on the North Eastern Uttar Pradesh and Western Madhya Pradesh of India respectively.

From thorough literature survey it was found that the only study on floristic diversity and ethnomedicinal uses of plant of Soor Sarovar Bird Sanctuary was done by Rani et al. (2009). So far no comprehensive study on the diversity of invasive alien plants is conducted on this region. The present study would be helpful for the academician, botanist and ecologist as basic information for future research towards conservation and management of the invasive alien plants of this region.

\section{Materials and methods}

\section{Study site}

A Keetham lake also known as Soor Sarovar National Bird Sanctuary which has been declared in 1991 Soor Sarovar Bird Sanctuary potential as a natural wonder has been recognised by the Uttar Pradesh forest department. Recently has been declared as National wetland in November 2007 by central forest and Ministry of environment (Gautam and Gautam, 2008). Keetham lake a place that ignited by passions of lord Krishna and Radha and inspired the famous poet Surdas to compose the Bhakti Kavya.

Soor Sarovar lake is located at a distance of $20 \mathrm{~km}$ from Agra city in Uttar Pradesh and a distance 180 Km from Delhi (Fig. 1). Keetham initially covering an area of 4.03 sq. $\mathrm{kms}$ has been expanded to an impressive area of 7.83 sq. kms. This pentagonal shaped lake's depth varies from $5 \mathrm{~m}$ to $8 \mathrm{~m}$. Soor Sarovar "Keetham Lake" from the riverine belt of the Yamuna River. Keetham also has wild life conservation in form of bear rescue facility and python point as well. Further this lake is surrounded by different kinds of wild vegetations. During the field survey it was observed that the natural wild vegetation is depleting day by day and in place Prosopis juliflora and Lantana camara are growing gregariously that posing a severe threat to the natural vegetation of Soor Sarovar Bird Sanctuary.

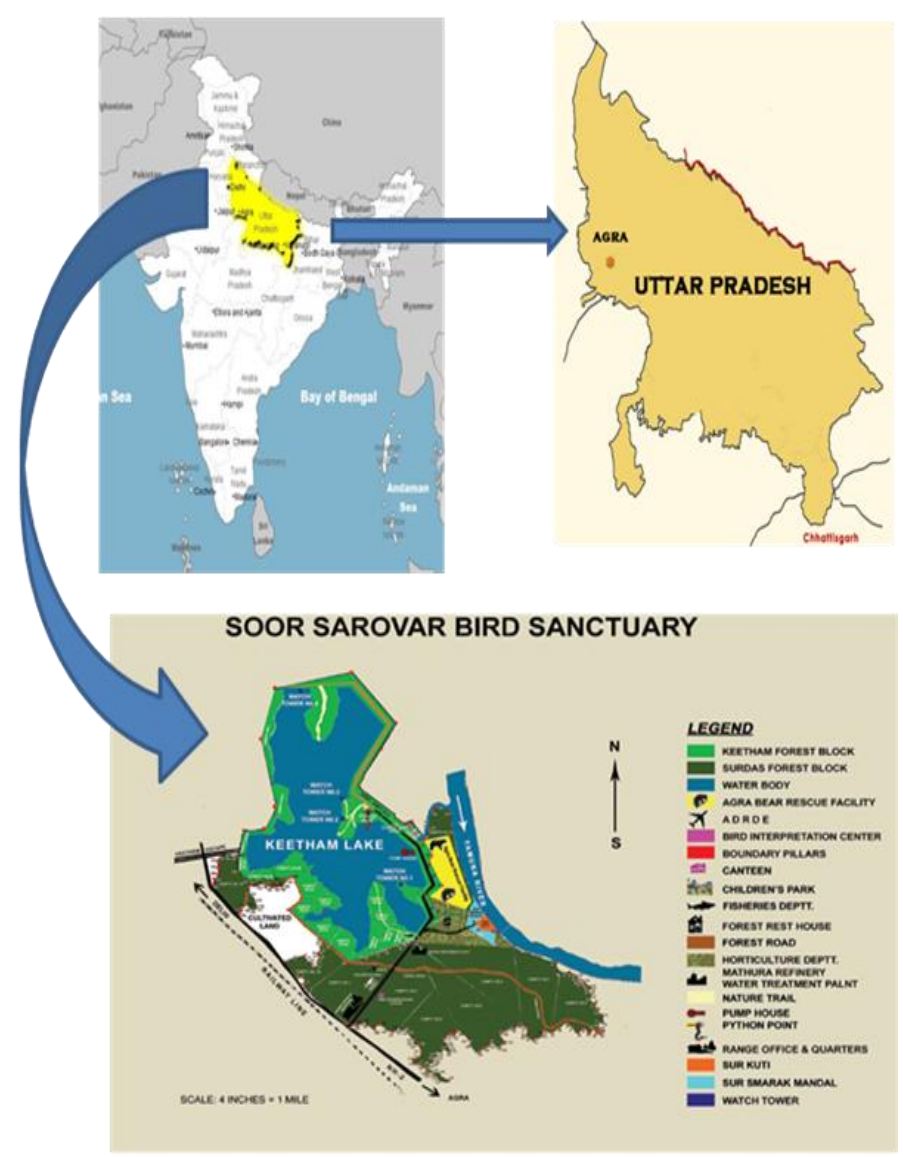

Fig. 1: Map of Soor Sarovar Bird Sanctuary.

\section{Field survey and data collection}

Extensive and intensive survey was undertaken for the collection of invasive alien plants in Soor Sarovar Bird Sanctuary during 2015. The collected specimens was processed for herbarium preparation by standard methods (Lawrence, 1951) and identified with the help of local floras and published literature (Hooker, 1822 1883; Cooke, 1901-1908; Duthie, 1903-1929; Gamble and Fisher, 1915-1936; Maheshwari, 1963; Verma et al., 1993, Khanna and Kumar, 2000, Khanna et al., 2001). The herbarium specimen was deposited in the herbarium of CSIR- National Botanical Research Institute, Lucknow. Information regarding nativity was collected from (Raghubanshi et al., 2005; Sujay et al., 2010; Singh, 1976; Sinha, 1976). The species are enumerated family wise alphabetically followed by botanical name, common name, life form, habitat, nativity, mode of introduction and categories. 


\section{Results and discussion}

A total 76 species distributed in 67 genera and 35 families recorded as invasive alien in Soor Sarovar Birad Sanctuary, Keetham. The family Asteraceae is the most dominant family with 12 species followed by Euphorbiaceae ( 7 species) Convolvulaceae $(6$ species), Solanaceae (5 species), Amaranthaceae (4 species) and Malvaceae, Mimosaceae and Poaceae (3 species each) (Fig. 2). These dominant families contributed $41 \%$ of the alien invasive flora of the Soor Sarovar Bird Sanctuary, Keetham. The genera with the highest number of alien species are Ipomoea (4 species), Alternanthera, Cassia, Chenopodium, Datura, Euphorbia and Jatropha (2 species each) (Table 1). These top genera contributed $21 \%$ taxa of alien flora of SSBS. Life form category shows that 56 species of herbs, 11 species of shrubs, 4 species of climbers, 4 species of trees and only one species in liana category (Fig. 3).
A total 11 different geographic region in terms of nativity are recorded in the present study. Among these (89\%) are contributed by the four major geographic region i.e., continent of America, Africa, Brazil and Europe. The American continent contributed majority (69\%) while Africa (10\%), Brazil and Europe (4\% each). Of the total alien plant species in SSBS 45 species are judged as naturalized, 21 interfering and 10 noxious (Fig.4). The most alarming condition is the number of noxious species that are harmful to the natural species. Further some of these species are known to be highly allergic and causing diseases in human being (Saxena, 1991 and Tripathi, 1999). Only 9 species namely Prosopis juliflora, Portulaca oleracea, Leucaena leucophloea, Lantana camara, Eichhornia crassipes, Duranta repens, Ageratum conyzoides, Chenopodium album and Celosia argentea have been introduced deliberately and rest of them unintentionally through trade exchange including grain import.

Table 1. List of Invasive plant species found in Soor Sarovar Bird Sanctuary, Keetham, Agra, India.

\begin{tabular}{|c|c|c|c|c|c|c|c|c|}
\hline Family & Botanical name & $\begin{array}{l}\text { Common } \\
\text { name }\end{array}$ & $\begin{array}{l}\text { Collection } \\
\text { number }\end{array}$ & $\begin{array}{l}\text { Life } \\
\text { form }\end{array}$ & Habitat & Nativity & $\begin{array}{l}\text { Mode of } \\
\text { introduction }\end{array}$ & Categories \\
\hline \multirow[t]{2}{*}{ Acanthaceae } & $\begin{array}{r}\text { Peristrophe paniculata } \\
\text { (Forssk.) Brummitt }\end{array}$ & $\begin{array}{l}\text { Panicled fold } \\
\text { wing }\end{array}$ & 258122 & Herb & W & $\begin{array}{l}\text { Tropical } \\
\text { America }\end{array}$ & $\mathrm{Ui}$ & Interfering \\
\hline & Ruellia tuberosa L. & Minnie root & 258120 & Herb & $\mathrm{AR}$ & $\begin{array}{l}\text { Tropical } \\
\text { America }\end{array}$ & $\mathrm{Ui}$ & Naturalized \\
\hline \multirow[t]{4}{*}{ Amaranthaceae } & $\begin{array}{l}\text { Alternanthera sessilis (L.) } \\
\text { R.Br. ex DC. }\end{array}$ & Joy weed & 258101 & Herb & $\mathrm{RB}$ & $\begin{array}{l}\text { Tropical } \\
\text { America }\end{array}$ & $\mathrm{Ui}$ & Naturalized \\
\hline & $\begin{array}{l}\text { Alternanthera philoxeroides } \\
\text { (Mart.) Griseb. }\end{array}$ & Alligator weed & 258077 & Herb & $\mathrm{W}$ & $\begin{array}{l}\text { Tropical } \\
\text { America }\end{array}$ & $\mathrm{Ui}$ & Naturalized \\
\hline & Celosia argentea $\mathrm{L}$. & $\begin{array}{l}\text { Silver cock's } \\
\text { comb }\end{array}$ & 258104 & Herb & $\mathrm{CF}$ & $\begin{array}{l}\text { Tropical } \\
\text { Africa }\end{array}$ & $\mathrm{Fd}$ & Naturalized \\
\hline & Gomphrena celosioides Mart. & $\begin{array}{l}\text { Bachelor's } \\
\text { button }\end{array}$ & 258125 & Herb & $\mathrm{CF}$ & $\begin{array}{l}\text { Tropical } \\
\text { America }\end{array}$ & $\mathrm{Ui}$ & Naturalized \\
\hline Apocynaceae & $\begin{array}{l}\text { Calotropis procera (Aiton) R. } \\
\text { Br. }\end{array}$ & Rubber bush & 258092 & Shrub & $\mathrm{W}$ & $\begin{array}{l}\text { Tropical } \\
\text { Africa }\end{array}$ & $\mathrm{Ui}$ & Interfering \\
\hline Araceae) & Pistia stratiotes L. & Water cabbage & 258123 & Herb & & $\begin{array}{l}\text { Tropical } \\
\text { America }\end{array}$ & $\mathrm{Ui}$ & Naturalized \\
\hline Arecaceae & Borassus flabellifer L. & Sugar palm & 258130 & Tree & $\mathrm{W}$ & $\begin{array}{l}\text { Tropical } \\
\text { Africa }\end{array}$ & $\mathrm{Ui}$ & Naturalized \\
\hline Asclepiadaceae & $\begin{array}{l}\text { Cryptostegia grandiflora } \\
\text { R.Br. }\end{array}$ & Rubber vine & 258134 & Liana & $\mathrm{CF}$ & Madagascar & $\mathrm{Ui}$ & Interfering \\
\hline \multirow[t]{9}{*}{ Asteraceae } & $\begin{array}{l}\text { Acanthospermum hispidum } \\
\text { DC. }\end{array}$ & Bristly starbur & 258114 & Herb & $\mathrm{W}$ & Brazil & $\mathrm{Ui}$ & Naturalized \\
\hline & Ageratum conyzoides L. & Chick weed & 258090 & Herb & $\mathrm{W}$ & $\begin{array}{l}\text { Tropical } \\
\text { America }\end{array}$ & $\mathrm{O}$ & Noxious \\
\hline & Blumea obliqua (L.) Druc & $\begin{array}{l}\text { Clustered } \\
\text { blumea }\end{array}$ & 258118 & Herb & $\mathrm{W}$ & $\begin{array}{l}\text { Tropical } \\
\text { America }\end{array}$ & $\mathrm{Ui}$ & Interfering \\
\hline & Echinops echinatus Roxb. & $\begin{array}{l}\text { Indian globe } \\
\text { thistle }\end{array}$ & 258103 & Herb & $\mathrm{W}$ & Afghanistan & $\mathrm{Ui}$ & Naturalized \\
\hline & Eclipta prostrata (L.) L. & False daisy & 258140 & Herb & AR & $\begin{array}{l}\text { Tropical } \\
\text { America }\end{array}$ & $\mathrm{Ui}$ & Naturalized \\
\hline & Lagascea mollis Cav. & $\begin{array}{l}\text { American } \\
\text { softhead }\end{array}$ & 258124 & Herb & $\mathrm{AR}, \mathrm{CF}$ & $\begin{array}{l}\text { Tropical Cent. } \\
\text { America }\end{array}$ & $\mathrm{Ui}$ & Noxious \\
\hline & Parthenium hysterophorus L. & Congress weed & 258106 & Herb & W & $\begin{array}{l}\text { Tropical North } \\
\text { America }\end{array}$ & $\mathrm{Ui}$ & Noxious \\
\hline & Sonchus oleraceus L. & Sow thistle & 258152 & Herb & $\mathrm{RB}$ & Mediterranean & $\mathrm{Ui}$ & Interfering \\
\hline & $\begin{array}{l}\text { Synedrella nodiflora (L.) } \\
\text { Gaertn. }\end{array}$ & Node weed & 258144 & Herb & $\mathrm{W}, \mathrm{AR}$ & West Indies & $\mathrm{Ui}$ & Naturalized \\
\hline
\end{tabular}




\begin{tabular}{|c|c|c|c|c|c|c|c|c|}
\hline & Tridax procumbens $\mathrm{L}$. & Coat button & 258116 & Herb & $\mathrm{CF}$ & $\begin{array}{l}\text { Tropical Cent. } \\
\text { America }\end{array}$ & $\mathrm{Ui}$ & Naturalized \\
\hline & Gnaphalium polycaulon Pers. & $\begin{array}{l}\text { Many } \\
\text { stemmed } \\
\text { cudweed }\end{array}$ & 258105 & Herb & $\mathrm{W}$ & $\begin{array}{l}\text { Tropical } \\
\text { America }\end{array}$ & $\mathrm{Ui}$ & Interfering \\
\hline Cactaceae & Opuntia elatior Mill. & Prickly pear & 258157 & Shrub & $\mathrm{AR}, \mathrm{W}$ & $\begin{array}{l}\text { Tropical } \\
\text { America }\end{array}$ & Ui & Noxious \\
\hline Caesalpiniaceae & Cassia tora $\mathrm{L}$. & Sickle senna & 258154 & Herb & W & $\begin{array}{l}\text { Tropical South } \\
\text { America }\end{array}$ & $\mathrm{Ui}$ & Noxious \\
\hline Capparidaceae & Cleome viscosa $\mathrm{L}$. & $\begin{array}{l}\text { Asian spidar } \\
\text { flower }\end{array}$ & 258159 & Herb & W & $\begin{array}{l}\text { Tropical } \\
\text { America }\end{array}$ & $\mathrm{Ui}$ & Naturalized \\
\hline \multirow[t]{2}{*}{ Chenopodiaceae } & Chenopodium album $\mathrm{L}$. & Goose foot & 258100 & Herb & $\mathrm{CF}$ & Europe & Fd & Interfering \\
\hline & Chenopodium murale $\mathrm{L}$. & $\begin{array}{l}\text { Australian } \\
\text { spinach }\end{array}$ & 258086 & Herb & $\mathrm{CF}, \mathrm{W}$ & $\begin{array}{l}\text { Tropical } \\
\text { America }\end{array}$ & $\mathrm{Ui}$ & Naturalized \\
\hline \multirow{4}{*}{ Convolvulaceae } & Ipomoea carnea Jacq. & $\begin{array}{l}\text { Bush morning } \\
\text { glory }\end{array}$ & 258160 & Shrub & W & $\begin{array}{l}\text { Tropical } \\
\text { America }\end{array}$ & Ui & Interfering \\
\hline & Ipomoea hederifolia $\mathrm{L}$. & $\begin{array}{l}\text { Scarlet } \\
\text { morning glory }\end{array}$ & 258131 & Climber & $\mathrm{F}$ & $\begin{array}{l}\text { Tropical } \\
\text { America }\end{array}$ & Ui & Interfering \\
\hline & Ipomoea nil (L.) Roth & $\begin{array}{l}\text { Ivy morning } \\
\text { glory }\end{array}$ & 258098 & Climber & FE, W & North America & Ui & Interfering \\
\hline & Ipomoea pestigridis $\mathrm{L}$. & $\begin{array}{l}\text { Tiger foot } \\
\text { morning glory }\end{array}$ & 258128 & Climber & W & $\begin{array}{l}\text { Tropical East } \\
\text { Africa }\end{array}$ & $\mathrm{Ui}$ & Interfering \\
\hline Cuscutaceae & Cuscuta reflexa Roxb. & Beggar weed & 258062 & Climber & $\mathrm{P}$ & Mediterranean & Ui & Interfering \\
\hline \multirow[t]{2}{*}{ Euphorbiaceae } & Ricinus communis $\mathrm{L}$. & $\begin{array}{l}\text { Castor bean } \\
\text { plant }\end{array}$ & 258079 & Tree & $\mathrm{W}, \mathrm{CF}$ & Africa & $\mathrm{Ui}$ & Interfering \\
\hline & $\begin{array}{l}\text { Chrozophora rottleri } \\
\text { (Geiseler) A.Juss. ex } \\
\text { Spreng. }\end{array}$ & $\begin{array}{l}\text { Rottler's } \\
\text { chrozophora }\end{array}$ & 258119 & Herb & W & Tropical & & Interfering \\
\hline Fabaceae & $\begin{array}{l}\text { Indigofera linifolia (L.f.) } \\
\text { Retz. }\end{array}$ & $\begin{array}{l}\text { Narrowleaf } \\
\text { indigo }\end{array}$ & 258126 & Herb & $\mathrm{AR}$ & $\begin{array}{l}\text { Tropical South } \\
\text { America }\end{array}$ & $\mathrm{Ui}$ & Naturalized \\
\hline \multirow[t]{2}{*}{ Lamiaceae } & Hyptis suaveolens (L.) Poit. & Chinese mint & 258117 & Herb & AR & $\begin{array}{l}\text { Tropical } \\
\text { America }\end{array}$ & $\mathrm{Ui}$ & Interfering \\
\hline & Осітит americanum L. & Common basil & 258082 & Herb & $\mathrm{W}$ & $\begin{array}{l}\text { Tropical } \\
\text { America }\end{array}$ & $\mathrm{Ui}$ & Naturalized \\
\hline Liliaceae & Asphodelus tenuifolius Cav. & Onion weed & 258127 & Herb & A & Trop. America & Ui & Interfering \\
\hline \multirow[t]{3}{*}{ Malvaceae } & $\begin{array}{l}\text { Malvastrum } \\
\text { coromandelianum (L.) } \\
\text { Gar. }\end{array}$ & Broom weed & 258094 & Herb & $\mathrm{W}$ & $\begin{array}{l}\text { Tropical } \\
\text { America }\end{array}$ & $\mathrm{Ui}$ & Naturalized \\
\hline & Sida acuta Burm. $f$. & $\begin{array}{l}\text { Common wire } \\
\text { weed }\end{array}$ & 258091 & Herb & $\mathrm{W}$ & $\begin{array}{l}\text { Tropical } \\
\text { America }\end{array}$ & $\mathrm{Ui}$ & Naturalized \\
\hline & Urena lobata $\mathrm{L}$. & Caesar weed & 258149 & Shrub & $\mathrm{W}$ & $\begin{array}{l}\text { Tropical } \\
\text { Africa }\end{array}$ & $\mathrm{Ui}$ & Interfering \\
\hline Martyniaceae & Martynia annua L. & Devil's claw & 258107 & Herb & W & $\begin{array}{l}\text { Tropical } \\
\text { America }\end{array}$ & $\mathrm{Ui}$ & Naturalized \\
\hline \multirow{3}{*}{ Mimosacceae } & $\begin{array}{l}\text { Prosopis juliflora (Swartz) } \\
\text { DC. }\end{array}$ & Mesquite & 258108 & Tree & $\mathrm{W}$ & Mexico & Af & Naturalized \\
\hline & $\begin{array}{l}\text { Leucaena leucocephala } \\
\text { (Lamk.) de Wit }\end{array}$ & White popinac & 258109 & Tree & W & $\begin{array}{l}\text { Tropical } \\
\text { America }\end{array}$ & Fo & Noxious \\
\hline & Mimosa pudica L. & Touch-me-not & 258155 & Herb & $\mathrm{F}$ & Brazil & $\mathrm{Ui}$ & Naturalized \\
\hline
\end{tabular}




\begin{tabular}{|c|c|c|c|c|c|c|c|c|}
\hline Onagraceae & $\begin{array}{l}\text { Ludwigia octovalvis (Jacq.) } \\
\text { Raven }\end{array}$ & $\begin{array}{l}\text { Mexican } \\
\text { primrose } \\
\text { willow }\end{array}$ & 258148 & Herb & $\mathrm{RB}$ & $\begin{array}{l}\text { Tropical } \\
\text { America }\end{array}$ & $\mathrm{Ui}$ & Naturalized \\
\hline Oxalidaceae & Oxalis corniculata $\mathrm{L}$. & Indian sorrel & 258095 & Herb & $\mathrm{CF}$ & Europe & Ui & Naturalized \\
\hline Papaveraceae & Argemone mexicana L. & Mexican poppy & 258099 & Herb & W & $\begin{array}{l}\text { Tropical South } \\
\text { America }\end{array}$ & Ui & Noxious \\
\hline Piperaceae & $\begin{array}{l}\text { Peperomia pellucida (L.) } \\
\text { Kunth }\end{array}$ & $\begin{array}{l}\text { State pencil } \\
\text { plant }\end{array}$ & 258139 & Herb & $\mathrm{AR}$ & $\begin{array}{l}\text { Tropical South } \\
\text { America }\end{array}$ & Ui & Naturalized \\
\hline \multirow[t]{2}{*}{ Poaceae } & Chloris barbata $\mathrm{Sw}$. & $\begin{array}{l}\text { Swollen mind } \\
\text { mill grass }\end{array}$ & 258080 & Herb & W & $\begin{array}{l}\text { Tropical } \\
\text { America }\end{array}$ & Ui & Naturalized \\
\hline & Saccharum spontaneum $\mathrm{L}$. & Kans grass & 258147 & Herb & $\mathrm{RB}$ & $\begin{array}{l}\text { Tropical West } \\
\text { Asia }\end{array}$ & $\mathrm{Ui}$ & Interfering \\
\hline Pontederiaceae & $\begin{array}{l}\text { Eichhornia crassipes (Mart.) } \\
\text { Solms }\end{array}$ & Water hyacinth & 258111 & Herb & A & $\begin{array}{l}\text { Tropical } \\
\text { America }\end{array}$ & $\mathrm{O}$ & Naturalized \\
\hline Portulacaceae & Portulaca oleracea $\mathrm{L}$. & Purslane & 258137 & Herb & W & $\begin{array}{l}\text { Tropical S. } \\
\text { America }\end{array}$ & $\mathrm{Fd}$ & Naturalized \\
\hline \multirow{4}{*}{ Solanaceae } & Datura metel L. & Devil's trumpet & 258112 & Shrub & W & $\begin{array}{l}\text { Tropical } \\
\text { America }\end{array}$ & Ui & Interfering \\
\hline & $\begin{array}{l}\text { Nicotiana plumbaginifolia } \\
\text { Viv. }\end{array}$ & $\begin{array}{l}\text { Tex-mex } \\
\text { tobacco }\end{array}$ & 258133 & Herb & W & $\begin{array}{l}\text { Tropical } \\
\text { America }\end{array}$ & Ui & Naturalized \\
\hline & Physalis minima $\mathrm{L}$. & Ground cherry & 258129 & Herb & W & $\begin{array}{l}\text { Tropical } \\
\text { America }\end{array}$ & $\mathrm{Ui}$ & Naturalized \\
\hline & Solanum nigrum L. & $\begin{array}{l}\text { Black } \\
\text { nightshade }\end{array}$ & 258113 & Herb & $\mathrm{CF}$ & $\begin{array}{l}\text { Tropical } \\
\text { America }\end{array}$ & $\mathrm{Ui}$ & Naturalized \\
\hline Sterculiaceae & Waltheria indica $\mathrm{L}$. & $\begin{array}{l}\text { Sleepy } \\
\text { morning }\end{array}$ & 258115 & Herb & $\mathrm{F}$ & $\begin{array}{l}\text { Tropical } \\
\text { America }\end{array}$ & $\mathrm{Ui}$ & Interfering \\
\hline Tiliaceae & Corchorus tridens L. & Fodder jute & 258146 & Herb & $\mathrm{AR}, \mathrm{W}$ & $\begin{array}{l}\text { Tropical } \\
\text { Africa }\end{array}$ & Ui & Naturalized \\
\hline
\end{tabular}

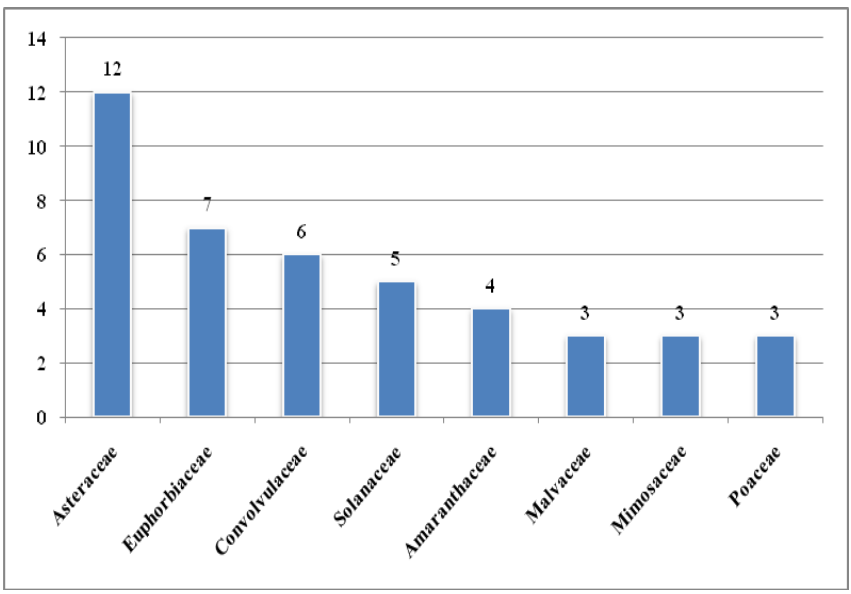

Fig. 2: Dominant families of Invasive alien plant species in SSBS.
Lantana camara and Prosopis juliflora are the most dominant species that acquired a large geographic area of the Soor Sarovar Bird Sanctuary. Lantana camara was first introduced in 1809 in Calcutta Botanical Garden for ornamental purpose (Thakur et al., 1992; Kannan et al., 2013). Now days it occurs all over the India ranging from tropical, subtropical and temperate region of the country (Kannan et al., 2013). There are several factors responsible for the successful invasion of the species like uncontrolled growth, absence of herbivores and pathogen (Keane and Crawley, 2002). Lantana camara is very efficient at nutrient uptake (Bhat et al., 1994) because of this potential it grow luxuriantly even in nutrient poor soil.

Prosopis juliflora was first introduced in between 1857 and 1878 in Thar desert of Northwest India used as a 
fuel. Prosopis juliflora has shown to be tolerant to draught and salinity (Pasiecznik et al., 2001) and also shows low palatability. Prosopis juliflora invasion speed is very high, due to this there is a reduction in fodder plants for wild as well as rearing cattle such as goat. They feed on the fruit of Prosopis juliflora in absence of the fodder plant that leads into the dispersal of the seed in surrounding areas. These are the factors are responsible for the successful invasion of the species and that affect the local biodiversity.

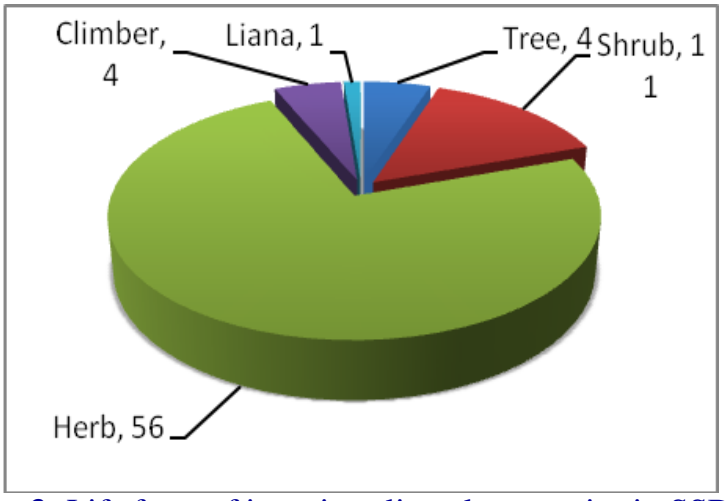

Fig. 3: Life form of invasive alien plant species in SSBS.

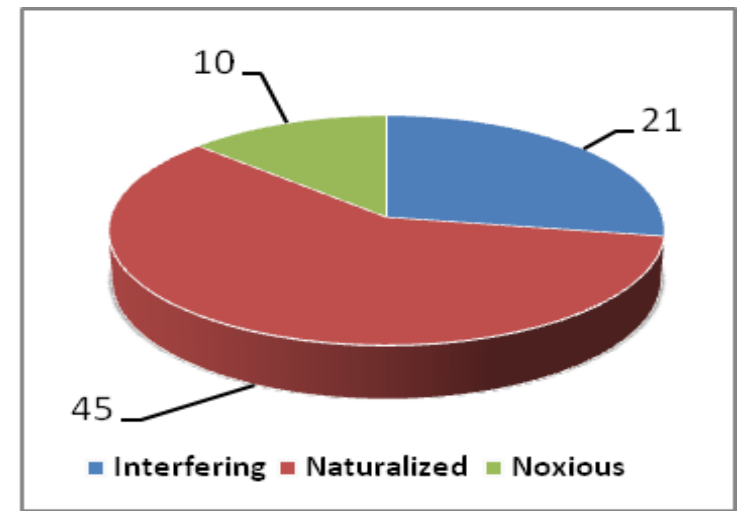

Fig. 4: Status of invasive alien plant species in SSBS.

The present study reports the dominant life form category is herbaceous species and it represent $(73 \%)$ of the total invasive alien plants. The factor greater viability and withstand in adverse condition helps herbaceous species in invasion though out the SSBS. Invasive species of Asteraceae exhibited a much higher reproductive capacity than those of other families. This high reproductive potential is achieved by partitioning of reproductive capital into a large number of propagules that are minute, light, and wind dispersed (Saxena and Ramakrishnan, 1982) similar result was observed by other workers in different parts of India such as (Rao and Murugan, 2006) found that the Asteraceae is dominating family in alien flora of India, in Uttar Pradesh
(Singh et al., 2010), in Indian Himalayan region (Sekar, 2012), in Johrat, Assam (Das and Duarah, 2013), in North eastern Uttar Pradesh (Srivastava et al., 2014) and in Western Madhya Pradesh (Wagh and Jain, 2015). Biological invasions of alien plants present one of the most serious threats to the indigenous biological diversity. Invasive alien plants have caused extensive economic and ecological damage throughout the world. In India Ageratum conyzoides, Parthenium hysterophorus, Lantana camara and Eupatorium adenophorum are major invaders and causing huge loss to indigenous species diversity in this part of the world (Dogra et al., 2009). Likewise invasive plant species like Ageratum conyzoides, Lantana camara, Prosopis juliflora, Parthenium hysterophorus, etc. are poses a major threat to indigenous biological diversity of Soor Sarovar Bird Sanctuary too.

\section{Conclusion}

The vegetation of Uttar Pradesh is known for its great diversity. Uttar Pradesh is one of the richest biodiversity zones of India. Therefore study on the impact of alien invasive plants on native phytodiversity of this region and their management is a need of hour. The invasive alien species possess serious threat to local biodiversity, affects crop production and human health. Therefore systematic study about the invasive plant species is required to provide adequate knowledge of the ecological and environmental consequences caused by invasive alien species and how to address it.

\section{Conflict of interest statement}

Authors declare that they have no conflict of interest.

\section{Acknowledgement}

The author is thankful to Director, CSIR- National Botanical Research Institute Lucknow, for encouragement and providing facilities to carry out the work. The study has been carried under in house project OLP-0083.

\section{References}

Bhatt, Y.D., Rawat, Y.S., Singh, S.P., 1994. Changes in ecosystem functioning after replacement of forest by Lantana shrubland in Kumaun Himalaya. J. Veg. Sci. 5, 67-70.

Cooke, T., 1901-1908. Flora of Bombay Presidency (Ed.: Singh, B., Singh, M.P.). Dehradun, India. 
Corlett, R.T., 1998. The naturalized flora of Singapore. J. Biogeogr. 15, 657-663.

Das, K., Duarah, P., 2013. Invasive alien plant species in the roadside areas of Johrat, Assam: Their harmful effects and beneficial uses. J. Engg. Res. Appl. 3(5), 353-358.

Dogra, K.S., Kohli, R.K, Sood, S.K., 2009. An assessment and impact of three invasive species of Himanchal Pradesh, India. Int. J. Biodiv. Conser. 1, 4-10.

Duthie, J.F., 1903-1922. Flora of Upper Gangetic Plain and the Adjacent of Siwalik and Sub-Himalayan Tracts. Vols. 1-3. Botanical Survey of India, Calcutta.

Enmoto, T., 1999. Naturalized weeds from foreign countries into Japan. In: Biological Invasions of Ecosystem by Pests and Beneficial Organisms (Eds.: Yano, E., Matsuo, K., Shiyomi, M., Andow, D. A.). National Institute of AgroEnvironmental Science, Tsukuba, Japan. 1-14.

Gamble, G.S., Fischer, C.F.C., 1957. Flora of Presidency of Madras. Vols. 1-3. Botanical Survey of India, Calcutta, India.

Gautam, R., Gautam, R.K., 2008. Variation in Physiochemical characteristic in Sur Sarovar. J. Natcon. 20(2), 343-348.

Hooker, J.D., 1822-1883. The Flora of British India. 7 Vols. L. Reeve \& Co., London.

Kannan, R., Shackleton, C.M., Uma Shaanker, R., 2013. Reconstructing the history of introduction and spread of the invasive species, Lantana, at three spatial scales in India. Biol. Invasions. 15, 1287-1302.

Keane, R.M., Crawley, M.J., 2002. Exotic plant invasions and the enemy release hypothesis. Trends Ecol. Evol. 17, 164170.

Khanna, K.K., Kumar, A., 2000. Additions to the taxa of Angiosperms: Flora of Madhya Pradesh Vol. II. J. Econ. Tax. Bot. 24, 177-180.

Khanna, K.K., Kumar, A., Dixit, R.D., Singh, N.P., 2001. Supplement to the Fora of Madhya Pradesh. Botanical Survey of India, Calcutta.

Kshirsagar, S.R., 2005. Origin, present status and distribution of exotic plants in South Gujarat. Ind. J. Forestr. 28, 136143.

Lawrence, G.H.M., 1951. Taxonomy of Vascular Plants. Macmillan, New York.

Maheshwari, J.K., 1963. The Flora of Delhi. Gossain \& Co. Pvt. Ltd., Calcutta.

McGeoch, M.A., Butchart, S.H.M., Spear, D., Marais, E., Kleynhans, E.J., Symes, A., Chanson, J., Hoffmann, M., 2010. Global indicators of biological invasion: Species numbers, biodiversity impact and policy responses. Diver. Distrib. 16(1), 95-108.

Meyer, J.Y., 2000. Preliminary review of the invasive plants in the Pacific islands. In: Invasive Species in the Pacific: A Technical Review and Draft Regional Strategy (Ed.: Sherley, G.). South Pacific Regional Environmental Programme, Samoa. pp.85-114.

Mooney, H.A., Hobbs, R.J., 2000. Invasive Species in a Changing World. Island Press, Washington, DC, USA.

Negi, P.S., Hajra, P.K., 2007. Alien flora of Doon Valley, Northwest Himalaya. Curr. Sci. 92(7), 968-978.
Pandey, R.P., Parmar, P.J., 1994. The exotic flora of Rajasthan. J. Econ. Taxon. Bot. 18, 105-121.

Pasiecznik, N.M., Felker, P., Harris, P.J.C., et al. 2001. The Prosopis juliflora-Prosopis pallida complex: a monograph. HDRA, Coventry Proceedings of a Conference held at the Central Arid Zone Research Institute, Jodhpur, Rajasthan, India. November 21-23, 1993. The Prosopis Society of India and The Henry Doubleday Research Association. pp.17-20.

Py̌sek, P., S'adlo, J., Mand'ak, B., 2002. Catalogue of alien plants of the Czech Republic. Preslia. 74(2), 97-186.

Raghubansh, A.S., Rai, L.C., Gaur, J.P., Singh, J.S., 2005. Invasive alien species and biodiversity in India. Curr. Sci. 88(4), 539-540.

Rani, R., Gautam, R., Gautam, R.K., 2009. Floristic survey of medicinal plants in Sur Sarovar wet land, Keetham, Agra India. J. Appl. Nat. Sci. 1(2), 196-200.

Rao, R.R., Murugan, R., 2006. Impact of exotic adventives weeds on native biodiversity in India: Implications for Conservation. In: Invasive Alien Species and Biodiversity in India (Eds.: Rai, L.C., Gaur, J.P.). Banaras Hindu University, Varanasi. pp.93-109.

Richardson, D.M., Py̌sek, P., Rejm'anek, M., Barbour, Dane Panetta, M.G. F., West, C.J., 2000. Naturalization and invasion of alien plants: concepts and definitions. Diver. Distrib. 6(2), 93-107.

Sawarker, V.B., 1984. Lantana camara on wildlife habitats with special reference to the Melaghat Tiger Reserve. Cheetal. 26, 24-38.

Saxena, K.G., 1991. Biological invasion in the Indian subcontinent: review of invasion by plants. In: Ecology of Biological Invasion in the Tropics (Ed.: Ramakrishnan, P.S.). International Scientific Publications, New Delhi, India. pp.53-73.

Saxena, K.G., Ramakrishnan, P.S., 1982. Partitioning of biomass and nutrients in the secondary successional herbaceous population subsequent to slash and burn. Proc. Indian Nat. Sci. Acad. 8, 807-818.

Sekar, K.C., 2012. Invasive alien plants of Indian Himalayan region diversity and implication. Amer. J. Plant Sci. 3, 177-184.

Singh, K.P., Shukla, A. N., Singh, J.S., 2010. State level inventory of invasive alien plants, their source regions and use potential. Curr. Sci. 99(1), 107-114.

Singh, P., 1976. Lantana weed and Lantana lace bug. Ind. Forestr. 102, 474-478.

Sinha, P.M., 1976. Studies on the use of some weedicides on Lantana camara. Ind. Forestr. 102, 298-305.

Srivastava, S., Dvivedi, A., Shukla, R.P., 2014. Invasive alien species of terrestrial vegetation of north eastern Uttar Pradesh. Int. J. For. Res. Article ID 959875, 9p. doi: $10.1155 / 2014 / 959875$.

Sujay, Y.H., Sattagi, H.N., Patil, R.K., (2010). Invasive alien insects and their impact on agroecosystem. Karnataka J. Agric. Sci. 23, 26-34.

Thakur, M.L., Ahmad, M., Thakur, R.K., 1992. Lantana weed (Lantana camara var. aculeata inn.) and its possible 
management through natural insect pests in India. Ind. Forestr. 118, 466-486.

Tripathi, S., 1999. Plant diversity of grassland of north-eastern U.P. with emphasis on population of Parthenium hysterophorus L. (Ph.D. thesis), Gorakhpur University, Gorakhpur, India.

Verma, D.M., Balakrishnan, N.P., Dixit, R.D., 1993. Flora of Madhya Pradesh - Vol. I. Botanical Survey of India, Calcutta.
Wagh, V.V., Jain, A.K., 2015. Invasive alien flora Jhabua district, Madhya Pradesh, India. Int. J. Biodiver. Conserv. 7(4), 227-237.

Wu, S. H., Hsieh, C. F., Rejm'anek, M., 2004. Catalogue of the naturalized flora of Taiwan. Taiwania. 49, 16-31.

\section{How to cite this article:}

Wagh, V. V., 2016. Diversity of invasive alien plants in Soor Sarovar Bird Sanctuary (SSBS), Keetham, Agra, India. Int. J. Curr. Res. Biosci. Plant Biol. 3(3), 62-69.

doi: http://dx.doi.org/10.20546/ijcrbp.2016.303.012 